\title{
Computed tomography pulmonary angiography for acute pulmonary embolism: prediction of adverse outcomes and 90 -day mortality in a single test
}

\author{
Neda Akhoundi ${ }^{1 A, B, D, E, F}$, Taraneh Faghihi Langroudii ${ }^{1 A, B}$, Hamid Rajebii ${ }^{2 E}$, Sepideh Haghi ${ }^{1 E}$, Mersede Paraham ${ }^{1 C}$, \\ Sonia Karami ${ }^{1 \mathrm{E}}$, Fatemeh Kheiridoust Langroudi ${ }^{\mathrm{B}}$
}

'Department of Radiology, Modarres Hospital, School of Medicine, Shahid Beheshti University of Medical Sciences, Tehran, Iran ¿University of Texas Health at San Antonio, TX, United States

\section{Abstract}

Purpose: Pulmonary embolism (PE) is a potentially fatal cardiopulmonary disease; therefore, rapid risk stratification is necessary to make decisions of appropriate management strategies. The aim of this study was to assess various computed tomography (CT) findings in order to find new prognostic factors of adverse outcome and mortality.

Material and methods: The study enrolled 104 patients with acute PE. Based on their outcome, patients were categorised into four groups. Comorbidities such as ischaemic heart disease were obtained from their medical records. Patients CT angiography were reviewed for recording variables such as main pulmonary artery diameter and right ventricle (RV)/left ventricle (LV) ratio. Patient deaths up to three months since diagnosis of PE had been registered. Logistic regression analysis was performed to find predictors.

Results: Based on multiple logistic regression, RV/LV ratio, LV diameter, and right-sided pulmonary infarction are predictors of mortality in 30 days. An RV/LV ratio of 1.19 could successfully discriminate patients who died within 30 days and those who did not.

Conclusions: RV/LV ratio, LV diameter, right-sided pulmonary infarction, assessed with helical CT, can help predict 30-day mortality.

Key words: prognostic factors, mortality, embolism.

\section{Introduction}

Pulmonary embolism (PE) is a potentially fatal cardiopulmonary disease with a mortality rate ranging from $5 \%$ to more than $30 \%$, depending on the patient's haemodynamic status [1-4]. Patients with PE may have different outcomes and therefore require varying intensities of clinical care. Consequently, risk assessment is necessary to make proper management strategy decisions [5-7]. Patients at high risk of adverse outcomes must be admitted to intensive care units and undergo thrombolytic treatment, whereas hospital discharge or home treatment might be options for patients with a low probability of adverse outcomes [8,9]. Because PE may cause death after right ventricular failure and circulatory collapse, right ventricle (RV) dysfunction should be detected rapidly to identify patients who might benefit from fibrinolytic therapy [1]. Recently, computed tomography has enabled noninvasive PE diagnosis [10,11]. Every year, many patients undergo pulmonary computed tomography (CT) angiography and receive ionising radiation; therefore, it would be prudent to investigate as many CT findings as possible to identify

Correspondence address:

Dr. Neda Akhoundi, Department of Radiology, Modarres Hospital, School of Medicine, Shahid Beheshti University of Medical Sciences, Tehran, Iran,

e-mail: neda.akhoundi@sbmu.ac.ir

Authors' contribution:

A Study design · B Data collection · C Statistical analysis · D Data interpretation - E Manuscript preparation · F Literature search · G Funds collection 
new predictors of adverse outcomes and mortality in these patients, to support better treatment and closer follow-up if needed. Some studies [7,12-15] have assessed the validity of CT scoring systems for pulmonary artery clot load, and others have reported RV dysfunction-related CT findings [16-21]. However, few studies have investigated the relationships among all other CT angiography findings and patients' clinical outcomes. Therefore, we conducted a study in patients with PE to define the prognostic value of CT for adverse outcomes and mortality.

\section{Material and methods}

\section{Study population}

The local Ethics Committee approved our retrospective study. Informed consent was waived, and patients' data were reviewed from January 2015 to January 2017. The inclusion criteria were positive PE on CT angiography study and at least three months of post-diagnosis follow-up. The exclusion criteria were chronic pulmonary embolism, cardiogenic shock, and poor quality of CT images. In total 112 patients with PE were identified. We excluded six patients because of the poor quality of images and two patients because of cardiogenic shock; a total of 104 patients were enrolled in the study.

\section{Clinical outcomes}

According to the outcomes, patients were categorised into the following four groups: group 1 included patients who experienced adverse outcomes, defined as worse clinical conditions such as using vasopressors because of hypotension, respiratory failure, or mechanical ventilation [22,23]; group 2 included patients who died $<30$ days after diagnosis of PE; group 3 included patients who died 30-90 days after diagnosis; and group 4 included patients who survived and did not experience any adverse outcomes, denoted as the OTHERS group. In our study, we considered a patient to have died of PE-related causes if (a) the autopsy report, death certificate, or medical records indicated PE as a cause of death or (b) shock was the leading cause of death in the absence of other cardiopulmonary diseases $[24,25]$.

\section{Covariate collection}

Information about patients' characteristics and comorbidities were obtained from the medical records [26].

The pulmonary embolism severity index (PESI) was retrospectively calculated by summing the following scores: patient age (years), male sex (10 points), cancer (30 points), heart failure (10 points), chronic lung disease (10 points), pulse rate $\geq 110 / \mathrm{min}$ ( 20 points), systolic blood pressure $<100 \mathrm{~mm} \mathrm{Hg}$ (30 points), respiratory rate $\geq 30 / \mathrm{min}$ (20 points), temperature $<36^{\circ} \mathrm{C}$ (20 points), altered mental status (60 points), and arterial oxygen saturation $<90 \%$ (20 points).

Each patient's score corresponded to the following risk classes: $\leq 65$, class I (very low risk); 66-85, class II (low risk); 86-105, class III (intermediate risk); 106-125, class IV (high risk); and > 125, class V (very high risk) [27].

\section{Computed tomography pulmonary angiography studies}

Computed tomography pulmonary angiography (CTPA) examinations were conducted in the craniocaudal direction by 64-multislice CT scanners (Brilliance CT scanner, Philips Healthcare) at a teaching hospital. Images were obtained with a slice thickness of $0.9 \mathrm{~mm}$, reconstructed at $0.5 \mathrm{~mm}$ slice intervals. Intravenous administration of $120 \mathrm{ml}$ of iodinated contrast medium (Visipaque $320 \mathrm{mg}$ ) was performed at $5-6 \mathrm{ml} / \mathrm{s}$, timed by bolus tracking at the main pulmonary artery. The CT criterion for PE diagnosis was nonocclusive thrombus (central filling defect outlined by the contrast agent) or occlusive thrombus in normal-sized or enlarged vessels [14]. A four-chambered heart view was used, as shown by Quiroz et al. [28]; the maximum transverse diameters of the right and left ventricle were measured at the level of the mitral and tricuspid valve. The pulmonary trunk diameter was measured on a plane that showed the main pulmonary artery and its bifurcation, and the diameters of the right and left pulmonary artery (RPA and LPA) were measured at the same level [29]. The diameter of the aorta was determined at the middle third of the ascending part. The anteroposterior (AP) and transverse diameters of the superior vena cava (SVC) were determined at the level at which the azygos vein entered the SVC, and the AP and transverse diameters of the inferior vena cava (IVC) were measured at the level at which the IVC joined the right atrium. We measured the transverse diameter of the azygos vein at the location where it was facing the right tracheal wall and joined the SVC [30]. The following observations were also recorded: leftward interventricular septal bowing, pleural effusion, and pulmonary findings, including pulmonary infarction, sidedness of the thrombus and level of the thrombus in the pulmonary artery tree. A radiologist with six years of experience in reading thoracic $\mathrm{CT}$ scans reported the images, who was blinded to the clinical history [31].

\section{Degree of vascular obstruction}

The pulmonary artery obstruction index (PAOI) was calculated by using the scoring system of Qanadli et al. [14]. We categorised PAOI scores into five classes: $\leq 8$, class I; 8-16, class II; 16-24, class III; 24-32, class IV; and 32-40, class V.

\section{Transthoracic echocardiographic evaluation}

Transthoracic echocardiography, which was performed within 24 hours of PE diagnosis, was used for 
extracting information about pulmonary artery pressure $[23,32,33]$.

\section{Three-month follow-up}

All patient deaths up to three months after PE diagnosis were assessed by an independent committee that was given access to all patient data. The committee outlined whether a patient death should be due to PE or should be attributed to other causes [21].

\section{Statistical analysis}

The Kolmogorov-Smirnov test was used for a normal distribution of continuous variables. Data with a normal distribution, such as PESI, were expressed as mean values with standard deviation. Parameters without normal distribution, such as age, were expressed as a median (firstthird inter-quartile range). Continuous data were compared with Anova or Kruskal-Wallis tests.

Chi-square test was used for comparing discrete variables. To assess the correlation between variables, Spearman's rho and Pearson's tests were used [34]. Cut-off values for variables to detect mortality, determined by receiver-operating characteristic (ROC) plots and areas under the curve, were calculated. We also calculated the threshold value for which sensitivity equalled specificity for measurements with an $\mathrm{A}_{\mathrm{z}}$ value significantly different from 0.5 [35]. Multiple logistic regression analyses were used to identify the predictors of adverse outcomes by using variables $p$-value $<0.05$ in univariate analysis. All statistical analyses were performed in SPSS version 24, SPSS Inc., Chicago, IL, USA.

\section{Results}

We included 104 consecutive patients with PE in the study. Patients' demographic and clinical characteristics are shown in Table 1. Sixteen patients experienced adverse outcomes. In three months, 15 deaths occurred. An independent adjudication committee concluded that all deaths could have been related to PE. Ten deaths occurred $<30$ days after diagnosis, and five occurred between days 30 and 90 .

The mean RV/left ventricle (LV) ratio was 1.11 (0.891.39), and the death within 30 days group had the highest ratio (1.44 [1.16-1.68]), followed by the adverse outcome group (1.2 [0.95-1.61]) $(p=0.024)$.

The frequency of right-sided pulmonary infarction was higher among the death within 30 days group (5 [50\%]) and adverse outcome group (5 [31\%]), and the lowest frequency was found in the OTHERS group (7 [9\%]) $(p=0.005)$.

The mean IVC AP diameter was $24 \mathrm{~mm}$ (21-28), and the adverse outcome group (20 mm [18-24]), death 30-90 days group (20 mm [16-29]), and death within 30 days group (23 mm [20-28]) had higher scores than the OTHERS group (25 mm [22-28]) $(p=0.017)$.
The azygos vein diameter was higher in the OTHERS group (10 mm [8-11]), with a $p$-value of 0.028 . The OTHERS group had more subsegmental artery involvement (52 [71\%]) than the adverse outcome (5 [31\%]), death within 30 days (4 [40\%]), and death within 30-90 days (1 [20\%]) groups $(p=0.003)$.

For the correlations between each of these three parameters (PAOI/PAOI class, PESI/PESI class, RV/LV, RV/LV class) and other variables (Table 2), Pearson's correlation and Spearman's rho test were performed. The correlation coefficients were highest between RV/LV and PAOI/ PAOI class and were lower (but still significant) between the SVC transverse diameter and PAOI/PAOI class. We found a negative correlation between the LV short-axis and PAOI $(-0.363)(p<0.001)$ and a positive correlation between MPA diameter and PESI.

In assessing the correlation between the RV/LV and other variables, the analysis revealed a significant correlation between leftward ventricular septal bowing and the $\mathrm{RV} / \mathrm{LV}$ ratio. Results showed a negative correlation between right sidedness of thrombus and the RV/LV ratio (Table 2).

\section{Predictors of outcomes at three months}

Based on univariate logistic regression analysis, significant baseline predictors of adverse outcome were PESI (OR 1.01; 95\% CI: 1.00-1.03; $p=0.031$ ), IVC AP diameter (mm) (OD 1.08; 95\% CI: $1-1.17 ; p=0.050)$, and right sidedness of the thrombus (OR 4.68; 95\% CI: 1.15-19.09; $p=0.031$ ), and the RV/LV ratio was a predictor of death within 30 days (OR 9.2; 95\% CI: 1.35-63.02; $p=0.023$ ). The results showed that right-sided pulmonary infarction (OR 0.127; 95\% CI: 0.03-0.535; $p=0.005$ ), bilateral pulmonary infarction (OR 0.241; 95\% CI: 0.06-0.976; $p=0.046$ ), ischaemic heart disease (IHD) (OD 0.150; 95\% CI: 0.036$0.624 ; p=0.009$ ), or heart failure (HF) (OR 0.135; 95\% CI: $0.027-0.677 ; p=0.015)$ were associated with a decreased risk of death within 30 days. Again, we found that a larger LV short-axis diameter (OR 0.089; 95\% CI: 0.797-0.993; $p=0.038)$ was associated with a decreased risk of death in this period. IVC transverse diameter ( $\mathrm{mm}$ ) (OR 0.786; 95\% CI: $0.630-0.981 ; p=0.033$ ) and chronic obstructive pulmonary disease (COPD) (OR 0.063; 95\% CI: 0.008$0.491 ; p=0.008$ ) were predictors of mortality in $30-90$ days, both of which showed a protective role against mortality in this period.

PAOI did not show a significant association with any groups $(p>0.05)$. Multivariable logistic regression analysis indicated that PESI (OR 1.01; 95\% CI: 1.00-1.03; $p=0.046)$ and right sidedness of the thrombus in the pulmonary tree (OR 4.5; 95\% CI: $1.01-19.96 ; p=0.048$ ) were predictors of adverse outcomes. Based on multiple logistic regression, the RV/LV ratio (OR 11.54; 95\% CI: 1.16$114.35 ; p=0.037)$ was a strong predictor of death within 30 days. The results showed that a longer LV short axis $(\mathrm{mm})(\mathrm{OR} 0.813$; 95\% CI: 0.681-0.969; $p=0.021$ ) or having 
Table 1. Comparisons of quantitative and qualitative characteristics of patients with pulmonary embolism between subgroups of mortality and adverse outcome

\begin{tabular}{|c|c|c|c|c|c|c|}
\hline Object & $\begin{array}{l}\text { All patients } \\
n=104\end{array}$ & $\begin{array}{l}\text { Adverse outcome } \\
\qquad n=16\end{array}$ & $\begin{array}{c}\text { Death }<30 \text { days } \\
n=10\end{array}$ & $\begin{array}{c}\text { Death } 30-90 \text { days } \\
\qquad n=5\end{array}$ & $\begin{array}{l}\text { Others } \\
n=73\end{array}$ & $p$-value \\
\hline $\begin{array}{l}\text { Age, years, median } \\
\left(25-75^{\text {th }} \text { percentiles }\right)\end{array}$ & $62.5(48-74)$ & $54(50-64)$ & $71(63-78)$ & $65(47-76)$ & $62(46-75)$ & 0.200 \\
\hline Male gender & $52(50 \%)$ & $7(43 \%)$ & $4(40 \%)$ & $4(80 \%)$ & $37(50 \%)$ & 0.482 \\
\hline PESI & $92 \pm 28$ & $109 \pm 34$ & $94 \pm 15$ & $95 \pm 8$ & $88 \pm 28$ & 0.057 \\
\hline PAOI & $10(3-20)$ & $10(4-23)$ & $15(9-26)$ & $11(5-17)$ & $8(3-19)$ & 0.247 \\
\hline MPA diameter (mm) & $29.4 \pm 3.8$ & $31.5 \pm 4.1$ & $26.5 \pm 3.1$ & $26.6 \pm 2.7$ & $29.6 \pm 3.6$ & 0.003 \\
\hline RPA diameter (mm) & $22(20-23)$ & $23(21-24)$ & $20(18-21)$ & $21(19-22)$ & $22(20-24)$ & 0.056 \\
\hline LPA diameter (mm) & $21(19-23)$ & $22(20-26)$ & $20(18-22)$ & $20(18-22)$ & $21(19-23)$ & 0.087 \\
\hline Aorta diameter (mm) & $32.8 \pm 4.2$ & $35.4 \pm 3.6$ & $33.4 \pm 3.9$ & $31.8 \pm 2.7$ & $32.3 \pm 4.3$ & 0.058 \\
\hline MPA/aorta ratio & $0.9 \pm 0.13$ & $0.89 \pm 0.10$ & $0.80 \pm 0.12$ & $0.84 \pm 0.13$ & $0.92 \pm 0.14$ & 0.039 \\
\hline RV short axis (mm) & $42.6 \pm 8.85$ & $44.33 \pm 6.59$ & $45.15 \pm 8.89$ & $41.86 \pm 7.22$ & $42.01 \pm 9.40$ & 0.625 \\
\hline LV short axis (mm) & $38.19 \pm 7.70$ & $37.21 \pm 6.98$ & $32.96 \pm 6.48$ & $43.08 \pm 3.37$ & $38.79 \pm 7.93$ & 0.06 \\
\hline RV/LV ratio & $1.11(0.89-1.39)$ & $1.2(0.95-1.61)$ & $1.44(1.16-1.68)$ & $0.88(0.83-1.17)$ & $1.03(0.88-1.35)$ & 0.024 \\
\hline SVC AP diameter (mm) & $22.52 \pm 4.41$ & $21.53 \pm 2.75$ & $23.10 \pm 5.97$ & $22.76 \pm 3.04$ & $22.65 \pm 4.58$ & 0.793 \\
\hline IVC AP diameter (mm) & $24(21-28)$ & $20(18-24)$ & $23(20-28)$ & $20(16-29)$ & $25(22-28)$ & 0.017 \\
\hline Azygos vein diameter & $9.6(8-11)$ & $9.1(8.27-11.5)$ & $7.6(6.37-10.22)$ & $8(6.5-8.9)$ & $10(8-11)$ & 0.028 \\
\hline Right sided pulmonary infarction & $18(17 \%)$ & $5(31 \%)$ & $5(50 \%)$ & $1(20 \%)$ & $7(9 \%)$ & 0.005 \\
\hline Left sided pulmonary infarction & $11(10 \%)$ & $1(6 \%)$ & $1(10 \%)$ & $1(20 \%)$ & $8(10 \%)$ & 0.849 \\
\hline \multicolumn{7}{|l|}{ Sidedness of thrombose } \\
\hline Right & $95(91 \%)$ & $16(100 \%)$ & $10(100 \%)$ & $4(80 \%)$ & $65(89 \%)$ & 0.288 \\
\hline Left & $92(88 \%)$ & $13(81 \%)$ & $10(100 \%)$ & $4(80 \%)$ & $65(89 \%)$ & 0.476 \\
\hline \multicolumn{7}{|l|}{ Level of thrombose } \\
\hline MPA & $3(2 \%)$ & $0(0 \%)$ & $0(0 \%)$ & $0(0 \%)$ & $3(4 \%)$ & 0.726 \\
\hline RPA & $15(14 \%)$ & $1(6 \%)$ & $2(20 \%)$ & $0(0 \%)$ & $12(16 \%)$ & 0.532 \\
\hline LPA & $11(10 \%)$ & $2(12 \%)$ & $2(20 \%)$ & $0(0 \%)$ & $7(9 \%)$ & 0.644 \\
\hline Lobar artery & $61(58 \%)$ & $10(62 \%)$ & $9(90 \%)$ & $4(80 \%)$ & $38(52 \%)$ & 0.094 \\
\hline Segmental artery & $88(84 \%)$ & $13(81 \%)$ & $10(100 \%)$ & $5(100 \%)$ & $60(82 \%)$ & 0.362 \\
\hline Subsegmental artery & $62(59 \%)$ & $5(31 \%)$ & $4(40 \%)$ & $1(20 \%)$ & $52(71 \%)$ & 0.003 \\
\hline PAP & $35(31-50)$ & $33.5(25-57)$ & $45.5(34.5-60)$ & $31(24-52)$ & $37(32-50)$ & 0.153 \\
\hline
\end{tabular}

PESI - pulmonary embolism severity index, PAOI - pulmonary artery obstruction index, MPA - main pulmonary artery, RPA - right pulmonary artery, LPA - left pulmonary artery, RV - right ventricle, LV - left ventricle, SVC - superior vena cava, IVC - inferior vena cava, PAP - pulmonary artery pressure

right-sided pulmonary infarction (OR $0.111 ; 95 \% \mathrm{CI}$ : $0.017-0.730 ; p=0.022$ ) was associated with a decreased risk of mortality within 30 days, and IVC transverse diameter $(\mathrm{mm})(\mathrm{OR} 749 ; 95 \%$ CI: 0.574-0.979; $p=0.034)$ and COPD (OR 0.041; 95\% CI: 0.004-0.448; $p=0.009$ ) were associated with decreased risk of death within 30-90 days (Table 3).

Measurable variables, such as pulmonary artery pressure (PAP), PESI, and main pulmonary artery (MPA) diameter, were tested for their ability to predict survival. $\mathrm{A}_{\mathrm{z}}$ was significantly higher than 0.5 for PESI $\left(\mathrm{A}_{\mathrm{z}}=0.667\right)$, MPA diameter $\left(\mathrm{A}_{\mathrm{z}}=0.663\right)$, LPA diameter $\left(\mathrm{A}_{\mathrm{z}}=0.683\right)$, and aorta diameter $\left(A_{z}=0.639\right)(p=0.035,0.039,0.021$, and 0.014 , respectively). We also detected an $\mathrm{A}_{\mathrm{z}}$ value of 0.744 and $p=0.011$ for the RV/LV ratio in the death within 30 days group (Table 4). For the factors which $\mathrm{A}_{\mathrm{z}}$ was significantly higher than 0.5 , Table 4 lists the threshold values for which the sensitivity equalled the specificity.

\section{Discussion}

The study investigated the prognostic value of pulmonary CT angiography findings in patients with symptomatic PE. Below, we discuss our findings separately based on the variables that correlated with mortality or adverse outcomes. 
Table 2. Correlation between pulmonary artery clot load, pulmonary embolism severity index, right ventricle/left ventricle, and other quantitative and qualitive computed tomography findings

\begin{tabular}{|c|c|c|c|c|c|c|}
\hline $\begin{array}{l}\text { Measurement and CT scan } \\
\text { findings }\end{array}$ & $\begin{array}{c}\text { PAOI/PAOI class } \\
\text { Correlation } \\
\text { coefficient }\end{array}$ & $p$-value & $\begin{array}{c}\text { PESI/PESI class } \\
\text { Correlation } \\
\text { coefficient }\end{array}$ & $p$-value & $\begin{array}{l}\text { RV/LV, RV/LV class } \\
\text { Correlation } \\
\text { coefficient }\end{array}$ & $p$-value \\
\hline PESI & 0.149 & 0.131 & - & - & 0.337 & $<0.001$ \\
\hline PAOI & - & - & 0.149 & 0.131 & 0.548 & $<0.001$ \\
\hline MPA diameter (mm) & 0.043 & 0.667 & 0.404 & $<0.001$ & 0.204 & 0.038 \\
\hline RPA diameter (mm) & -0.042 & 0.673 & 0.499 & $<0.001$ & $22(20-24)$ & 0.056 \\
\hline LPA diameter (mm) & 0.066 & 0.507 & 0.469 & $<0.001$ & $21(19-23)$ & 0.087 \\
\hline Aorta diameter (mm) & 0.000 & 0.996 & 0.361 & $<0.001$ & 0.158 & 0.109 \\
\hline MPA/Aorta ratio & 0.040 & 0.683 & 0.044 & 0.657 & 0.028 & 0.777 \\
\hline RV short axis (mm) & 0.426 & $<0.001$ & 0.369 & $<0.001$ & - & - \\
\hline LV short axis (mm) & -0.363 & $<0.001$ & -0.084 & 0.397 & - & - \\
\hline RV/LV ratio & 0.548 & $<0.001$ & 0.337 & $<0.001$ & - & - \\
\hline SVC transverse diameter $(\mathrm{mm})$ & 0.206 & 0.036 & 0.353 & $<0.001$ & 0.300 & 0.002 \\
\hline IVC AP diameter (mm) & -0.130 & 0.188 & 0.193 & 0.049 & -0.122 & 0.217 \\
\hline IVC transverse diameter (mm) & 0.010 & 0.920 & 0.265 & 0.007 & 0.244 & 0.012 \\
\hline Leftward ventricular septal bowing & -0.124 & 0.210 & 0.198 & 0.044 & 0.433 & $<0.001$ \\
\hline Reflux into inferior vena cava & -0.158 & 0.109 & 0.194 & 0.048 & 0.170 & 0.085 \\
\hline Right-sided pleural effusion & -0.120 & 0.224 & -0.040 & 0.688 & -0.072 & 0.466 \\
\hline Left-sided pleural effusion & -0.133 & 0.179 & 0.095 & 0.336 & -0.071 & 0.474 \\
\hline Bilateral pleural effusion & -0.025 & 0.805 & 0.095 & 0.339 & -0.232 & 0.018 \\
\hline Right-sided MGG opacity & 0.055 & 0.579 & 0.040 & 0.689 & -0.153 & 0.121 \\
\hline Left-sided MGG opacity & -0.154 & 0.119 & 0.203 & 0.039 & -0.031 & 0.754 \\
\hline \multicolumn{7}{|l|}{ Sidedness of thrombose } \\
\hline Right & 0.049 & 0.619 & 0.020 & 0.841 & -0.348 & $<0.001$ \\
\hline left & 0.095 & 0.335 & -0.009 & 0.925 & 0.079 & 0.423 \\
\hline \multicolumn{7}{|l|}{ Level of thrombose } \\
\hline MPA & 0.012 & 0.903 & -0.008 & 0.937 & 0.260 & 0.008 \\
\hline RPA & -0.081 & 0.414 & 0.021 & 0.835 & 0.258 & 0.008 \\
\hline$\underline{L P A}$ & -0.002 & 0.987 & 0.024 & 0.808 & 0.259 & 0.008 \\
\hline Lobar artery & 0.089 & 0.369 & 0.156 & 0.113 & 0.368 & $<0.001$ \\
\hline Segmental artery & 0.069 & 0.483 & 0.089 & 0.369 & 0.261 & 0.007 \\
\hline Subsegmental artery & 0.062 & 0.531 & -0.089 & 0.371 & -0.303 & 0.002 \\
\hline PAP & 0.306 & 0.002 & 0.247 & 0.011 & 0.194 & 0.049 \\
\hline
\end{tabular}

CT - computed tomography, PESI - pulmonary embolism severity index, PAOI - pulmonary artery obstruction index, MPA - main pulmonary artery, RPA - right pulmonary artery, LPA - left pulmonary artery, RV - right ventricle, LV - left ventricle, SVC - superior vena cava, IVC - inferior vena cava, PAP - pulmonary artery pressure

\section{Main pulmonary artery diameter ( $\mathrm{mm}$ )}

Our study showed that the maximum MPA diameter (31.5 $\pm 4.1)$ in the adverse outcome group was significantly meaningful, a finding similar to the study performed by Ilker et al. [29] (31.3, 95\% CI: 24.3-46.4) in patients who experienced adverse outcomes; however, their findings did not reach significance $(p=0.209)$. In another study done by Lyhne et al. [7], increased diameter of the MPA
$(\mathrm{OR}=1.08$ per $1 \mathrm{~mm}$ increase, $p=0.047)$ was associated with severe adverse outcome. Collomb et al. [16] found that patients with severe pulmonary embolism have larger central pulmonary artery diameters than those in other patients; we had similar findings. According to the study done by Beenen et al. [6], MPA enlargement was significantly associated with mortality in both the short and long term (OR 4.18 [95\% CI: 1.04-16.76] at 1 week to OR 2.33 [95\% CI: 1.36-3.97] after one year). 
Table 3. Predictors of adverse outcome and mortality in 30 days and 30-90 days of 104 patients with acute pulmonary embolism

\begin{tabular}{|c|c|c|c|c|c|c|}
\hline Risk factor & $\begin{array}{l}\text { Adverse outcome } \\
\text { Univariable } \\
\text { analysis } \\
\text { OR }(95 \% \mathrm{Cl}) \\
\text { p-value }\end{array}$ & $\begin{array}{c}\text { Multivariable } \\
\text { analysis } \\
\text { OR }(95 \% \mathrm{CI}) \\
p \text {-value }\end{array}$ & $\begin{array}{l}\text { Death }<30 \text { days } \\
\text { Univariable } \\
\text { analysis } \\
\text { OR }(95 \% \mathrm{Cl}) \\
p \text {-value }\end{array}$ & $\begin{array}{c}\text { Multivariable } \\
\text { analysis*** } \\
\text { OR }(95 \% \mathrm{Cl}) \\
p \text {-value }\end{array}$ & $\begin{array}{l}\text { Death } 30-90 \text { days } \\
\text { Univariable } \\
\text { analysis } \\
\text { OR }(95 \% \mathrm{Cl}) \\
\text { p-value }\end{array}$ & $\begin{array}{c}\text { Multivariable } \\
\text { analysis } \\
\text { OR }(95 \% \mathrm{CI}) \\
p \text {-value }\end{array}$ \\
\hline Age, per year & $\begin{array}{c}1(0.97-1.03) \\
0.873\end{array}$ & - & $\begin{array}{c}1.04(0.995-1.102) \\
0.076\end{array}$ & - & $\begin{array}{c}0.997(0.945-1.05) \\
0.919\end{array}$ & - \\
\hline Male gender & $\begin{array}{c}0.9(0.36-2.21) \\
0.819\end{array}$ & - & $\begin{array}{c}2.1(0.503-9.02) \\
0.304\end{array}$ & - & $\begin{array}{c}0.235(0.025-2.18) \\
0.203\end{array}$ & - \\
\hline PESI & $\begin{array}{c}1.01(1-1.03) \\
0.031\end{array}$ & $\begin{array}{c}1.01(1-1.03) \\
0.046\end{array}$ & $\begin{array}{c}0.998(0.974-1.02) \\
0.885\end{array}$ & - & $\begin{array}{c}1.00(0.973-1.03) \\
0.804\end{array}$ & - \\
\hline PAOI & $\begin{array}{c}1.01(0.96-1.06) \\
0.694\end{array}$ & - & $\begin{array}{c}1.07(0.996-1.16) \\
0.062\end{array}$ & - & $\begin{array}{c}0.992(0.892-1.1) \\
0.888\end{array}$ & - \\
\hline $\begin{array}{l}\text { MPA diameter } \\
(\mathrm{mm})\end{array}$ & $\begin{array}{c}0.994(0.88-1.11) \\
0.922\end{array}$ & - & $\begin{array}{c}0.824(0.678-1) \\
0.051\end{array}$ & - & $\begin{array}{c}0.809(0.627-1.04) \\
0.104\end{array}$ & - \\
\hline $\begin{array}{l}\text { RPA diameter } \\
(\mathrm{mm})\end{array}$ & $\begin{array}{c}1.08(0.95-1.22) \\
0.201\end{array}$ & - & $\begin{array}{c}0.844(0.676-1.05) \\
0.133\end{array}$ & - & $\begin{array}{c}0.884(0.669-1.16) \\
0.387\end{array}$ & - \\
\hline $\begin{array}{l}\text { LPA diameter } \\
(\mathrm{mm})\end{array}$ & $\begin{array}{c}1.03(0.908-1.18) \\
0.585\end{array}$ & - & $\begin{array}{c}0.887(0.710-1.107) \\
0.288\end{array}$ & - & $\begin{array}{c}0.924(0.696-1.228) \\
0.587\end{array}$ & - \\
\hline $\begin{array}{l}\text { Aorta diameter } \\
(\mathrm{mm})\end{array}$ & $\begin{array}{c}1(0.90-1.11) \\
0.980\end{array}$ & - & $\begin{array}{c}0.980(0.834-1.152) \\
0.809\end{array}$ & - & $\begin{array}{c}0.939(0.759-1.16) \\
0.563\end{array}$ & - \\
\hline $\begin{array}{l}\text { MPA/aorta } \\
\text { ratio }\end{array}$ & $\begin{array}{c}0.984(0.038-25.53) \\
0.992\end{array}$ & - & $\begin{array}{c}0.012(0.00-2.39) \\
0.102\end{array}$ & - & $\begin{array}{c}0.033(0.00-26.63) \\
0.318\end{array}$ & - \\
\hline $\begin{array}{l}\text { RV short axis } \\
(\mathrm{mm})\end{array}$ & $\begin{array}{c}1.02(0.972-1.07) \\
0.388\end{array}$ & - & $\begin{array}{c}1.04(0.967-1.12) \\
0.280\end{array}$ & - & $\begin{array}{c}0.989(0.892-1.09) \\
0.834\end{array}$ & - \\
\hline $\begin{array}{l}\text { LV short axis } \\
(\mathrm{mm})\end{array}$ & $\begin{array}{c}1.00(0.95-1.07) \\
0.753\end{array}$ & - & $\begin{array}{c}0.89(0.797-0.993) \\
0.038\end{array}$ & $\begin{array}{c}0.813(0.681-0.969) \\
0.021^{*}\end{array}$ & $\begin{array}{c}1.08(0.969-1.21) \\
0.153\end{array}$ & - \\
\hline RV/LV ratio & $\begin{array}{c}1.31(0.35-4.9) \\
0.679\end{array}$ & - & $\begin{array}{c}9.2(1.35-63.02) \\
0.023\end{array}$ & $\begin{array}{c}11.54(1.16-114.35) \\
0.037^{* *}\end{array}$ & $\begin{array}{c}0.109(0.003-3.92) \\
0.226\end{array}$ & - \\
\hline $\begin{array}{l}\text { IVC AP } \\
\text { diameter (mm) }\end{array}$ & $\begin{array}{c}1.08(1-1.17) \\
0.050\end{array}$ & $\begin{array}{c}1.05(0.96-1.15) \\
0.262\end{array}$ & $\begin{array}{c}0.965(0.843-1.105) \\
0.608\end{array}$ & - & $\begin{array}{c}0.884(0.717-1.08) \\
0.244\end{array}$ & - \\
\hline $\begin{array}{l}\text { IVC transverse } \\
\text { diameter (mm) }\end{array}$ & $\begin{array}{c}0.971(0.894-1.05) \\
0.479\end{array}$ & - & $\begin{array}{c}0.943(0.827-1.075) \\
0.379\end{array}$ & - & $\begin{array}{c}0.786(0.630-0.981) \\
0.033\end{array}$ & $\begin{array}{c}0.749(0.574-0.979) \\
0.034\end{array}$ \\
\hline $\begin{array}{l}\text { Right-sided } \\
\text { pulmonary } \\
\text { infarction }\end{array}$ & $\begin{array}{c}2.9(0.62-13.7) \\
0.174\end{array}$ & - & $\begin{array}{c}0.127(0.03-0.535) \\
0.005\end{array}$ & $\begin{array}{c}0.111(0.017-0.730) \\
0.022^{*} \\
0.164(0.031-0.877) \\
0.035^{* *}\end{array}$ & $\begin{array}{c}0.829(0.087-7.88) \\
0.871\end{array}$ & - \\
\hline $\begin{array}{l}\text { Left-sided } \\
\text { pulmonary } \\
\text { infarction }\end{array}$ & $\begin{array}{c}1.4(0.29-7.34) \\
0.633\end{array}$ & - & $\begin{array}{c}0.941(0.106-8.323) \\
0.957\end{array}$ & - & $\begin{array}{c}0.449(0.046-4.42) \\
0.493\end{array}$ & - \\
\hline \multicolumn{7}{|l|}{$\begin{array}{l}\text { Sidedness } \\
\text { of thrombose }\end{array}$} \\
\hline Right & $\begin{array}{c}4.68(1.15-19.09) \\
0.031\end{array}$ & $\begin{array}{c}4.5(1.01-19.96) \\
0.048\end{array}$ & - & - & $\begin{array}{c}2.8(0.283-28.57) \\
0.375\end{array}$ & - \\
\hline Left & $\begin{array}{c}0.60(0.12-2.94) \\
0.529\end{array}$ & - & - & - & $\begin{array}{c}0.5(0.051-4.88) \\
0.551\end{array}$ & - \\
\hline
\end{tabular}


Table 3. Cont.

\begin{tabular}{|c|c|c|c|c|c|c|}
\hline Risk factor & $\begin{array}{l}\text { Adverse outcome } \\
\text { Univariable } \\
\text { analysis } \\
\text { OR }(95 \% \mathrm{Cl}) \\
\text { p-value }\end{array}$ & $\begin{array}{l}\text { Multivariable } \\
\text { analysis } \\
\text { OR }(95 \% \mathrm{CI}) \\
\text { p-value }\end{array}$ & $\begin{array}{c}\text { Death }<30 \text { days } \\
\text { Univariable } \\
\text { analysis } \\
\text { OR }(95 \% \mathrm{Cl}) \\
p \text {-value }\end{array}$ & $\begin{array}{c}\text { Multivariable } \\
\text { analysis*** } \\
\text { OR }(95 \% \mathrm{Cl}) \\
\text { p-value }\end{array}$ & $\begin{array}{c}\text { Death } 30-90 \text { days } \\
\text { Univariable } \\
\text { analysis } \\
\text { OR }(95 \% \mathrm{Cl}) \\
\text { p-value }\end{array}$ & $\begin{array}{l}\text { Multivariable } \\
\text { analysis } \\
\text { OR ( } 95 \% \mathrm{Cl}) \\
\text { p-value }\end{array}$ \\
\hline \multicolumn{7}{|l|}{$\begin{array}{l}\text { Level of } \\
\text { thrombose }\end{array}$} \\
\hline MPA & $\begin{array}{c}0.623(0.05-7.17) \\
0.705\end{array}$ & - & - & - & - & - \\
\hline RPA & $\begin{array}{c}0.407(0.129-1.28) \\
0.126\end{array}$ & - & $\begin{array}{c}0.555(0.104-2.96) \\
0.491\end{array}$ & - & - & - \\
\hline LPA & $\begin{array}{c}0.826(0.202-3.38) \\
0.791\end{array}$ & - & $\begin{array}{c}0.366(0.066-2.03) \\
0.251\end{array}$ & - & - & - \\
\hline IHD & $\begin{array}{c}0.93(0.303-2.90) \\
0.911\end{array}$ & - & $\begin{array}{c}0.150(0.036-0.624) \\
0.009\end{array}$ & $\begin{array}{c}0.310(0.05-1.928) \\
0.209^{*} \\
0.300(0.052-1.71) \\
0.176^{* *}\end{array}$ & $\begin{array}{c}0.950(0.1-8.99) \\
0.964\end{array}$ & - \\
\hline $\mathrm{HF}$ & $\begin{array}{c}2.7(0.321-22.75) \\
0.360\end{array}$ & - & $\begin{array}{c}0.135(0.027-0.677) \\
0.015\end{array}$ & $\begin{array}{c}0.161(0.013-2.04) \\
0.159^{*} \\
0.314(0.031-3194) \\
0.328^{* *}\end{array}$ & $\begin{array}{c}0.35(0.035-3.53) \\
0.375\end{array}$ & - \\
\hline COPD & $\begin{array}{c}0.613(0.105-3.56) \\
0.586\end{array}$ & - & - & - & $\begin{array}{c}0.063(0.008-0.491) \\
0.008\end{array}$ & $\begin{array}{c}0.041(0.004-0.448) \\
0.009\end{array}$ \\
\hline
\end{tabular}

***0nly variables found to significantly predict adverse outcome or mortality in 90 days by univariable analysis were entered in the multivariable model.

Two multivariable logistic regression models are used for the variables that are significantly meaningful in univariable analysis for death $<30$ days group, the first one that is shown by * includes rightsided pulmonary infarction, LV short axis (mm), IHD, and HF. The second one ** includes RV/LV, right-sided pulmonary infarction, IHD, and HF.

PESI - pulmonary embolism severity index, PAOI - pulmonary artery obstruction index, MPA - main pulmonary artery, RPA - right pulmonary artery, LPA - left pulmonary artery, RV - right ventricle, LV - left ventricle, SVC - superior vena cava, IVC - inferior vena cava, PAP - pulmonary artery pressure, IHD - ischaemic heart disease, HF - heart failure, COPD - chronic obstructive pulmonary disease

Our findings indicate a strong positive correlation between MPA diameter and PESI, but we did not observe a relationship between MPA diameter and PAOI, a finding that has been previously reported [30]. ROC analysis indicated that when the MPA diameter was 29.9, the sensitivity was equal to the specificity (i.e. $62.5 \%$ ) for discriminating between patients who did and did not experience adverse outcomes. A similar finding was observed in the previous study [18], which found that MPA diameter could identify patients with right ventricular dysfunction, with a cut-off value of $29 \mathrm{~mm}$. However, our study could not show the MPA diameter as predictive of mortality within 90 days, thus confirming the findings of a previous study [31], which was unable to demonstrate that a PA diameter $>30 \mathrm{~mm} \mathrm{Hg}$ is a predictor of mortality within 30 days or adverse outcomes.

\section{Main pulmonary artery/aorta ratio}

This ratio significantly differed among subgroups in our study; the death within 30 days group had the lowest range $(0.8 \pm 0.12)$, and the OTHERS group had the highest range $(0.92 \pm 0.14)$. These findings were similar to those from a previous study by Ilker et al. [29], which demonstrated that an MPA/aorta ratio of 0.78 (0.48-0.87) in patients with mortality within 30 days was the lowest among the studied population, but, contrary to our findings, the results were not significant $(p=0.101)$. Our study did not demonstrate that the MPA/aorta ratio is a predictor of adverse outcomes or mortality, in accordance with findings from three other studies [21,30,31] in which the diameter of MPA and the MPA/aorta ratio were not found to be indicators of the mortality or severity of acute PE. We did not find any correlation between MPA/ aorta and PAOI, in agreement with Ghaye et al. [30].

\section{Right ventricle/left ventricle ratio}

Patients with PE and pulmonary hypertension might have right ventricular enlargement, which can lead to elevated RV/LV ratios [23]. We found the highest ratio in adverse outcomes and death in the death within 30 days group, thereby supporting findings from previous studies $[21,36,37]$ showing a correlation between the RV/LV ratio and MDCT and mortality. Our study showed a strong positive relationship between $\mathrm{RV} / \mathrm{LV}$ and PAOI along 
Table 4. Performance of computed tomography and echocardiography measurements for discrimination of survivors from non-survivors (each mortality group), as indicated by receiver operating characteristic curves

\begin{tabular}{|c|c|c|c|c|c|}
\hline Measurement & $\begin{array}{c}\text { Adverse outcome } \\
A_{z} \text { value } \\
\text { Value* } \\
p \text {-value }\end{array}$ & $\begin{array}{c}\text { Death }<30 \text { days } \\
A_{z} \text { value } \\
\text { Value } \\
p \text {-value }\end{array}$ & $\begin{array}{c}\text { Death } 30-90 \text { days } \\
A_{z} \text { value } \\
\text { Value } \\
p \text {-value }\end{array}$ & $\begin{array}{l}\text { Threshold } \\
\text { value }^{* *}\end{array}$ & $\begin{array}{c}\text { Sensitivity nearly } \\
\text { equals specificity } \\
(\%)^{* *}\end{array}$ \\
\hline PAP & $\begin{array}{c}0.414 \\
(0.233,0.594) \\
0.274\end{array}$ & $\begin{array}{c}0.638 \\
(0.493,0.782) \\
0.153\end{array}$ & $\begin{array}{c}0.295 \\
(0.000,0.591) \\
0.123\end{array}$ & NA & NA \\
\hline PAOI & $\begin{array}{c}0.540 \\
(0.384,0.697) \\
0.608\end{array}$ & $\begin{array}{c}0.679 \\
(0.511,0.847) \\
0.063\end{array}$ & $\begin{array}{c}0.504 \\
(0.257,0.752) \\
0.976\end{array}$ & NA & NA \\
\hline PESI & $\begin{array}{c}0.667 \\
(0.513,0.820) \\
0.035 \\
\end{array}$ & $\begin{array}{c}0.551 \\
(0.410,0.692) \\
0.597 \\
\end{array}$ & $\begin{array}{c}0.583 \\
(0.447,0.719) \\
0.533 \\
\end{array}$ & 94.5 & 68.8 \\
\hline MPA diameter (mm) & $\begin{array}{c}0.663 \\
(0.510,0.816) \\
0.039\end{array}$ & $\begin{array}{c}0.254 \\
(0.101,0.408) \\
0.011\end{array}$ & $\begin{array}{c}0.261 \\
(0.077,0.444) \\
0.072\end{array}$ & 29.9 & 62.5 \\
\hline RPA diameter (mm) & $\begin{array}{c}0.652 \\
(0.521,0.782) \\
0.054\end{array}$ & $\begin{array}{c}0.312 \\
(0.161,0.464) \\
0.052 \\
\end{array}$ & $\begin{array}{c}0.371 \\
(0.205,0.537) \\
0.331\end{array}$ & NA & NA \\
\hline LPA diameter (mm) & $\begin{array}{c}0.683 \\
(0.540,0.825) \\
0.021\end{array}$ & $\begin{array}{c}0.381 \\
(0.226,0.536) \\
0.219\end{array}$ & $\begin{array}{c}0.408 \\
(0.191,0.625) \\
0.489\end{array}$ & 20.5 & 75 \\
\hline Aorta diameter $(\mathrm{mm})$ & $\begin{array}{c}0.693 \\
(0.563,0.823) \\
0.014\end{array}$ & $\begin{array}{c}0.528 \\
(0.353,0.704) \\
0.770\end{array}$ & $\begin{array}{c}0.418 \\
(0.235,0.602) \\
0.538\end{array}$ & 32.9 & 75 \\
\hline MPA/aorta ratio & $\begin{array}{c}0.466 \\
(0.334,0.599) \\
0.669 \\
\end{array}$ & $\begin{array}{c}0.281 \\
(0.128,0.434) \\
0.023 \\
\end{array}$ & $\begin{array}{c}0.310 \\
(0.052,0.568) \\
0.153 \\
\end{array}$ & NA & NA \\
\hline RV short axis (mm) & $\begin{array}{c}0.593 \\
(0.458,0.728) \\
0.238\end{array}$ & $\begin{array}{c}0.590 \\
(0.397,0.783) \\
0.351\end{array}$ & $\begin{array}{c}0.459 \\
(0.221,0.696) \\
0.755\end{array}$ & NA & NA \\
\hline LV short axis (mm) & $\begin{array}{c}0.465 \\
(0.316,0.614) \\
0.656 \\
\end{array}$ & $\begin{array}{c}0.280 \\
(0.132,0.428) \\
0.023\end{array}$ & $\begin{array}{c}0.719 \\
(0.587,0.852) \\
0.099 \\
\end{array}$ & NA & NA \\
\hline RV/LV ratio & $\begin{array}{c}0.587 \\
(0.431,0.743) \\
0.270\end{array}$ & $\begin{array}{c}0.744 \\
(0.600,0.888) \\
0.011\end{array}$ & $\begin{array}{c}0.328 \\
(0.132,0.524) \\
0.197\end{array}$ & 1.19 & 80 \\
\hline
\end{tabular}

$A_{z}$ values were calculated and tested for difference from 0.5 . For $A_{z}$ values that showed difference from 0.5 , threshold values were calculated for which sensitivity equalled specificity. *Data in parentheses are $95 \%$ confidence intervals. ${ }^{* *} \mathrm{NA}-$ not applicable.

PAP - pulmonary artery pressure, PAOI - pulmonary artery obstruction index, PESI - pulmonary embolism severity index, MPA - main pulmonary artery, RPA - right pulmonary artery, LPA - left pulmonary artery, RV - right ventricle, LV - left ventricle

with RV/LV and PESI; these findings are consistent with those from a previous study [38] that found a moderate to strong correlation between RV/LV and PAOI. ROC analysis indicated that an RV/LV ratio of 1.19 could successfully discriminate patients who die within 30 days from those who do not, with sensitivity equal to the specificity, at $80 \%$; however, this cut-off point is different from those in previous studies. Quiroz et al. [39] set a cut-off point of 0.9, and George et al. [26] described a cut-off point of 1 for this purpose. Moreover, our study demonstrated that the $\mathrm{RV} / \mathrm{LV}$ ratio is a strong predictor of mortality within 30 days, thus emphasising the benefit of calculating the $\mathrm{RV} / \mathrm{LV}$ ratio in patients with acute PE for determining prognosis [38]. This finding has also been shown in previous studies $[38,40]$.

\section{Inferior vena cava diameter}

Based on our findings, patients who experienced adverse outcomes or mortality within 90 days had the lowest 
IVC AP diameters, and the IVC transverse diameter had a weak positive correlation with PESI and the RV/LV ratio. Any increase in IVC transverse diameter was associated with a lower probability of death in 30-90 days. These results should be investigated in future larger trials.

\section{Right-sided pulmonary infarction}

Pulmonary infarction has been reported to be associated with a lower mortality rate $[40,41]$. Similarly, our results showed that right-sided pulmonary infarction (RSPI) decreased the mortality within 30 days in patients with PE. In accordance with findings from previous studies $[30,31,38]$, we did not find that pulmonary infarction was a predictor of adverse outcome and mortality within 90 days.

\section{Pulmonary embolism severity index}

We found a moderate positive correlation between PESI and the RV/LV ratio. According to our findings with a cut-off point of 94.5, PESI successfully discriminated patients with and without adverse outcomes, with sensitivity equal to the specificity, at $68.8 \%$.

We showed that PESI could be used as a predictor of adverse outcomes, in agreement with the findings of Choi et al. [27], in which PESI, NT-Pro BNP, and troponin were demonstrated to be prognostic factors of adverse outcomes.

\section{Pulmonary artery obstruction index}

We did not find any meaningful differences in PAOI among the groups, and our results indicate that PAOI cannot be used to predict mortality or adverse outcomes. However, our findings show a strong positive correlation between PAOI and the $\mathrm{RV} / \mathrm{LV}$ ratio.

\section{Aorta, right pulmonary artery, and left pulmonary artery diameter}

Our results showed that RPA, LPA, and aorta diameter had strong, strong, and moderate positive correlations, respectively, with PESI. With the cut-off points of $20.5 \mathrm{~mm}$ for LPA diameter and $32.9 \mathrm{~mm}$ for aorta diameter, we were able to discriminate patients with and without adverse outcomes, with sensitivity equal to the specificity of $75 \%$. According to previous studies [30], the aorta diameter is higher in non-survivors than in survivors (36.1 \pm 4.5 ); these findings were similar to ours, in which the aorta diameters in the adverse outcome group (35.4 \pm 3.6$)$ and the mortality within 30 days group $(33.4 \pm 3.9)$ were higher than those in the remaining groups.

\section{Right ventricle and left ventricle diameter}

$\mathrm{PE}$, along with pulmonary hypertension, may cause right ventricular enlargement. Our study showed that the RV and LV short-axis diameters did not differ among groups. Oz et al. [29] have reported similar findings, in which the LV diameter was not found to differ among patients with and without adverse clinical outcomes, and among patients who did and did not die within 30 days; however, there was a difference in RV diameter between patients with and without adverse clinical outcomes $(p=0.001)$.

According to our analysis, RV diameter has a strong positive correlation with PAOI, similarly to the findings of a previous study [30]. Moreover, there was a moderate positive correlation between RV diameter and PESI. Our results showed that any increase in PAOI makes the LV diameter decrease, in accordance with findings from a previous study [30] reporting a robust negative correlation between LV diameter and PAOI.

Our study demonstrated that any decrease in LV diameter increases the probability of death within 30 days and, therefore, might be used as a predictor of mortality.

\section{Superior vena cava transverse diameter}

Our study showed a weak positive correlation between SVC transverse diameter and PAOI, similar to the findings of a previous study [30] that did not show such a relation.

We also found a moderate positive correlation between SVC transverse diameter and PESI and the RV/LV ratio. In our analysis, the differences in the diameter of SVC among subgroups were not significantly meaningful, but Ghaye et al. [30] demonstrated that mean SVC diameter in non-survivors is higher than that in survivors; also, Ghuysen et al. [28] found a significant correlation of SVC diameter with mortality.

\section{Right sidedness of thrombus}

According to our findings, there was a moderate negative correlation between right sidedness of the thrombus and the RV/LV ratio: right sidedness of the thrombus was correlated with a lower RV/LV ratio and was found to be a predictor of adverse outcomes.

\section{Chronic obstructive pulmonary disease}

We found that patients with COPD were less likely to die in the 30 to 90 -day period, a new finding not reported in previous studies $[23,34,42]$.

\section{Limitations}

The present study also has some limitations. First, our PAOI (median 10.5, range 4-20) was relatively low. Second, the potential effects of PE-specific therapy on clinical outcomes were not considered. Third, the small group size (death < 30 days; death 30-90 days; adverse outcome) may bias the results. 


\section{Conclusions}

Our study is the first attempting to find correlations between various CT findings and adverse outcomes or mortality within 90 days in four subgroups of patients with PE. We determined that RV/LV, LV diameter, and right-sided pulmonary infarction are predictors of mortality within 30 days, and IVC diameter and COPD have prognostic value in determining mortality in the 30 to 90-day period. We also found that PESI and right sidedness of the thrombus are predictors of adverse outcomes.

\section{Conflict of interest}

The authors report no conflict of interest.

\section{References}

1. Goldhaber SZ, Visani L, De Rosa M. Acute pulmonary embolism: clinical outcomes in the International Cooperative Pulmonary Embolism Registry (ICOPER). Lancet 1999; 353: 1386-1389.

2. Quinlan DJ, McQuillan A, Eikelboom JW. Low-molecular-weight heparin campared with intravenous unfractionated heparin for treatment of pulmonary embolism: A meta-analysis of randomized, controlled trials. Ann Intern Med 2004; 140: 175-183.

3. Douketis JD, Kearon C, Bates S, et al. Risk of fetal pulmonary embolism in patients with treated venous thromboembolism. JAMA 1998; 279: 458-462.

4. Buller HR, Davidson BL, Decousus H, et al. Subcutaneous fondaparinux versus intravenous unfractionated heparin in the initial treatment of pulmonary embolism. N Engl J Med 2003; 349: 1695-1702.

5. Torbicki A, Perrier A, Konstantinides S, et al. Task Force for the Diagnosis and Management of Acute Pulmonary Embolism of the European Society of Cardiology (ESC). Guidelines on the diagnosis and management of acute pulmonary embolism. Eur Heart J 2008; 29: 2276-2315.

6. Beenen L, Bossuyt P, Stoker J, Middeldrop S. Prognostic value of cardiovascular parameters in computed tomography pulmonary angiography in patients with acute pulmonary embolism. Eur Respir J 2018; 52: pii: 1702611.

7. Lyhne MD, Schultz JG, MacMahon PJ, et al. Septal bowing and pulmonary artery diameter on computed tomography pulmonary angiography are associated with short-term outcomes in patients with acute pulmonary embolism. Emerg Radiol 2019; 26: 623-630.

8. Wan S, Quinlan DJ, Agnelli G, Eikelboom JW. Trombolysis compared with heparin for the initial treatment of initial treatment of pulmonary embolism: a meta-analysis of the randomized controlled trials. Circulation 2004; 110: 744-749.

9. Squizzato A, Galli M, Dentali F, Ageno W. Outpatient treatment and early discharge of symptomatic pulmonary embolism: a systematic review. Eur Respir J 2009; 33: 1148-1155.

10. Stein PD, Fowler SE, Goodman LR, et al. Multidetector computed tomography for acute pulmonary embolism. N Engl J Med 2006; 354: 2317-2327.

11. Zhang LJ, Lu GM, Meinel FG, et al. Computed tomography of acute pulmonary embolism: state-of-the-art. Eur Radiol 2015; 25: 2547 2557.
12. Vedovati MC, Germini F, Agenelli G, Becattini C. Prognostic role of embolic burden assessed at computed tomography angiography in patients with acute pulmonary embolism: systematic review and meta-analysis. J Thromb Haemost 2013; 11: 2092-2102.

13. Bankier AA, Janata K, Fleischmann D, et al. Severity assessment of acute pulmonary embolism with spiral CT: evaluation of two modified angiographic scores and comparison with clinical data. J Thoracic Imaging 1997; 12: 150-158.

14. Qanadli SD, El Hajjam M, Vieillard-Baron A, et al. New CT, index to quantify arterial obstruction in pulmonary embolism: comparison with angiographic index and echocardiography. AJR Am J Roentgenol 2001; 176: 1415-1420.

15. Mastora I, Remmy-Jardin M, Massen P, et al. Severity of acute pulmonary embolism: evaluation of a new spiral CT angiographic score in correlation with echocardiographic data. Eur Radiol 2003; 13: 29-35.

16. Collomb D, Paramelle PJ, Calaque O, et al. Severity assessment of acute pulmonary embolism: evaluation using helical CT. Eur Radiol 2003; 13: 1508-1514.

17. Araoz PA, Gotway MB, Trowbridge RL, et al. Helical CT pulmonary angiography predictors of in-hospital morbidity and mortality in patients with acute pulmonary embolism. J Thorac Imaging 2003; 18: 207-216.

18. Gutte H, Mortenson J, Mark ML, et al. Non-ECG-gated CT pulmonary angiography and the prediction of right ventricular dysfunction in patients suspected of pulmonary embolism. Clin Physiol Funct Imaging 2017; 37: 575-581.

19. Bach AG, Nansalmaa B, Kranz J, et al. CT pulmonary angiography findings that predict 30-day mortality in patients with acute pulmonary embolism. Eur J Radiol 2015; 84: 332-337.

20. Wu AS, Pezzullo JA, Cronan JJ, et al. CT pulmonary angiography :quantification of pulmonary embolus as a predictor of patient out-come-initial experience. Radiology 2004; 230: 831-835.

21. Van der Meer RW, Pattynama PM, Van Strijen MJ, et al. Right ventricular dysfunction and pulmonary obstruction index at helical CT: prediction of clinical outcome during 3-month follow-up in patients with acute pulmonary embolism. Radiology 2005; 235: 798-803.

22. Konstantinides S, Gebel A, Heusel G, et al. Heparin plus alteplase compared with heparin alone in patients with submassive pulmonary embolism. N Engl J Med 2002; 347: 1143-1150.

23. Jimenez D, Lobo JL, Monreal M, et al. Prognostic significance of multidetector CT in normotensive patients with pulmonary embolism: results of the PROTECT study. Thorax 2014; 69: 109-115.

24. Casazza F, Becattini C, Bongarzoni A, et al. Clinical features and short term outcomes of patients with acute pulmonary embolism. The Italian Pulmonary Embolism Registry (IPER). Thromb Res 2012; 130: 847-852.

25. Laporte S, Mismetti P, Decousus H, et al. Clinical predictors of fetal pulmonary embolism in 15,520 patients with venous thromboembolism: findings from the Registro Informatizado de la Enfermedad TromboEmbolica venosa (RIETE) Registry. Circulation 2008; 117: 1711-1716.

26. George E, Kumamaru KK, Ghosh N, et al. Computed tomography and echocardiography in patients with acute pulmonary embolism: part 2 prognostic value. J Thoracic Imaging 2014; 29: W7-W12. 
27. Keum-Ju C, Seung-Ick C, Kyung-Min S, et al. Prognostic implications of computed tomographic right ventricular dilation in patients with acute pulmonary embolism. Thromb Res 2013; 113: 182-186.

28. Ghuysen A, Ghaye B, Willems V, et al. Computed tomographic pulmonary angiography and prognostic significance in patients with acute pulmonary embolism. Thorax 2005; 60: 956-961.

29. Oz II, Altinsoy B, Serifoglu I, et al. Evaluation of right atrium-toright ventricle diameter ratio on computed tomography pulmonary angiography: prediction of adverse outcome and 30-day mortality. Eur J Radiol 2015; 84: 2526-2532.

30. Ghaye B, Ghuysen A, Willems V, et al. Severe pulmonary embolism: pulmonary artery clot load scores and cardiovascular parameters as predictors of mortality. Radiology 2006; 239: 884-891.

31. Sen HS, Abakay O, Cetincakmak MG, et al. A single imaging modality in the diagnosis, and prognosis of pulmonary embolism. Biomed Res Int 2014; 2014: 470295.

32. Grifoni S, Olivotto I, Cecchini P, et al. Short term clinical outcome of patients with pulmonary embolism, normal blood pressure and echocardiographic right ventricular dysfunction. Circulation 2000; 101: 2817-2822.

33. Sanchez O, Trinquart L, Caille V, et al. Prognostic factors for pulmonary embolism: the PREP study, a prospective multicenter cohort study. Am J Respir Crit Care Med 2010; 181: 168-173.

34. Ozsu S, Karaman K, Mentese A, et al. Combined risk stratification with computerized tomography/echocardiography and biomarkers in patients with normotensive pulmonary embolism. Tromb Res 2010; 126: 486-492.

35. Hanley JA, McNeil BJ. The meaning and use of the area under a receiver operating characteristic (ROC) curve. Radiology 1982; 143: 29-36.

36. Schoepf UJ, Kucher N, Kipfmueller F, et al. Right ventricular enlargement on chest computed tomography. Circulation 2004; 110: 32763280 .

37. Gibson NS, Sohne M, Buller HR. Prognostic value of echocardiography and spiral computed tomography in patients with pulmonary embolism. Curr Opin Pulm Med 2005; 11: 380-384.

38. Heyer CM, Lemburg SP, Knoop H, Holland T. Multidetector-CT angiography in pulmonary embolism - can image parameters predict clinical outcome? Eur Radiol 2011; 21: 1928-1937.

39. Quiroz R, Kucher N, Schoepf UJ, et al. Right ventricular enlargement on chest computed tomography: prognostic role in acute pulmonary embolism. Circulation 2004; 20: 2401-2404.

40. Tajarernmuang P, Euathrongchit J, Liwsrisakun C, et al. Computed tomographic pulmonary angio graphic findings to predict adverse outcomes in acute pulmonary embolism. Crit Care 2015; 19: 327.

41. Lobo JL, Zorrila V, Arizpuru F, et al. Clinical syndromes and clinical outcome in patients with pulmonary embolism: findings from the RIETE registry. Chest 2006; 130: 1817-1822.

42. Becattini C, Agnelli G, Vedovati MC, et al. Multidetector computed tomography for acute pulmonary embolism: diagnosis and risk stratification in a single test. Eur Heart J 2011; 32: 1657-1663. 